\title{
Primary Tibiotalocalcaneal Fusion for High-risk Complex Ankle Fractures
}

\author{
Nicholas Genovese ${ }^{1}$, Jay Patel ${ }^{2}$, Sheldon S Lin ${ }^{3}$, Joshua Greendyk ${ }^{4}$
}

\begin{abstract}
Successful management of high-risk diabetic patients with unstable ankle fractures remains a difficult task given the high incidence of complications and need for reoperation. Ankle arthrodesis has been primarily described as a salvage procedure for Charcot arthropathy or failed fracture fixation. Primary tibiotalocalcaneal (TTC) fusion is a simple and effective treatment option for dealing with the pathophysiologic factors that diabetic patients encounter when they sustain an unstable ankle fracture.

Keywords: Ankle arthrodesis, Ankle fractures, Diabetic ankle fracture, Fusion, Tibiotalocalcaneal arthrodesis.

Journal of Foot and Ankle Surgery (Asia Pacific) (2021): 10.5005/jp-journals-10040-1180
\end{abstract}

\section{INTRODUCTION}

Ankle fractures make up $9 \%$ of all adult fractures, with one in eight patients being diabetic. ${ }^{1-3}$ This number continues to grow annually as the prevalence of diabetes increases globally. ${ }^{4}$ Injuries range from low-energy rotational injuries to severe fracture-dislocations often with significant soft tissue compromise. While these injuries may be common, diabetic patients present a unique clinical challenge. Poor bone and soft-tissue quality as well as other host factors that further limit healing potential leading to increased risk of complications. Diabetic patients who sustain ankle fractures that require operative fixation have a complication rate of roughly $40 \%$, nearly 3 -fold higher than patients without diabetes. ${ }^{5}$ Wound breakdown, deep infection, nonunion, hardware failure, and Charcot arthropathy are all common complications that often necessitate repeat operations. ${ }^{6}$ As a result, treatment plans for diabetics may be guided by a greater consideration for soft-tissue injury, glucose control, and the presence of end-organ damage. As the incidence of both diabetes and ankle fractures continue to increase, it is increasingly important to determine an appropriate treatment protocol to limit complications and repeat procedures for these patients.

The difficulties and complications from diabetes stem from hyperglycemic states leading to arteriosclerosis, resulting in relatively hypoxic tissues with poorer collagen synthesis and immune cellularity. While comparing diabetic patients treated with open reduction and internal fixation (ORIF) for ankle fractures to non-diabetic cohorts, several studies have found that diabetic patients have worse outcomes. ${ }^{2,7-9}$ Studies have also shown several predictive risk factors for poor outcomes with conventional ORIF of ankle fractures, including open injuries, diabetes mellitus, peripheral neuropathy, and peripheral vascular disease. ${ }^{2}$ Wukich et al. found that diabetic patients with concomitant peripheral vascular disease who are forced to remain immobile after these injuries are at an even greater risk of perioperative complications. ${ }^{6}$

Current recommendations for operative treatment of ankle fractures in diabetic patients consist of increased rigid fixation in addition to increased periods of immobilization to avoid mechanical failure. Techniques implemented to achieve rigid fixation for highrisk ankle fractures include the use of locking plate constructs, combination internal and external fixation, multiple quadricortical

\begin{abstract}
${ }^{1-3}$ Department of Orthopaedics, Rutgers New Jersey Medical School, Newark, New Jersey, USA

${ }^{4}$ Undergraduate University, New Jersey Institute of Technology, Newark, New Jersey, USA

Corresponding Author: Nicholas Genovese, Department of Orthopaedics, Rutgers New Jersey Medical School, Newark, New Jersey, USA, Phone: +91 9082396938, e-mail:ng503@njms.rutgers.edu How to cite this article: Genovese N, Patel J, Lin SS, et al. Primary Tibiotalocalcaneal Fusion for High-risk Complex Ankle Fractures. J Foot Ankle Surg (Asia Pacific) 2021;8(4):188-192.
\end{abstract}

Source of support: Nil

Conflict of interest: None

syndesmotic screws, intramedullary fibular Kirschner wires, and augmentation of standard fixation with transarticular Steinmann pins or screws. ${ }^{10-12}$

One controversial treatment option lies in the concept of primary tibiotalocalcaneal (TTC) fusion with internal fixation after a diabetic ankle fracture. Use of tibiotalar or TTC fusion has traditionally been reserved for patients with Charcot arthropathy or failed fracture fixation. Described methods for TTC arthrodesis include TTC hindfoot nails, ringed external fixators, and TTC arthrodesis with crossed screws or plating. ${ }^{13}$ This article aims to stress the importance of operative rigid fixation of complicated ankle fractures in diabetic patients and to present primary TTC fusion as a viable alternative for dealing with the pathophysiologic factors that diabetic patients face.

\section{Case Description}

Our case begins with a 59-year-old woman with a history of ischemic stroke, type I diabetes mellitus, residual right hemiparesis, epilepsy, and previous right hip fracture fixation who presented with right ankle pain. X-ray examination demonstrated a fracture-dislocation of the right ankle with posterior subluxation of the talus relative to the tibia (Fig. 1). After a failed closed reduction attempt in the emergency department (Fig. 2), an ankle-spanning external fixation was applied by the on-call orthopedist on the night of presentation

(c) The Author(s). 2021 Open Access This article is distributed under the terms of the Creative Commons Attribution 4.0 International License (https://creativecommons. org/licenses/by-nc/4.0/), which permits unrestricted use, distribution, and non-commercial reproduction in any medium, provided you give appropriate credit to the original author(s) and the source, provide a link to the Creative Commons license, and indicate if changes were made. The Creative Commons Public Domain Dedication waiver (http://creativecommons.org/publicdomain/zero/1.0/) applies to the data made available in this article, unless otherwise stated. 


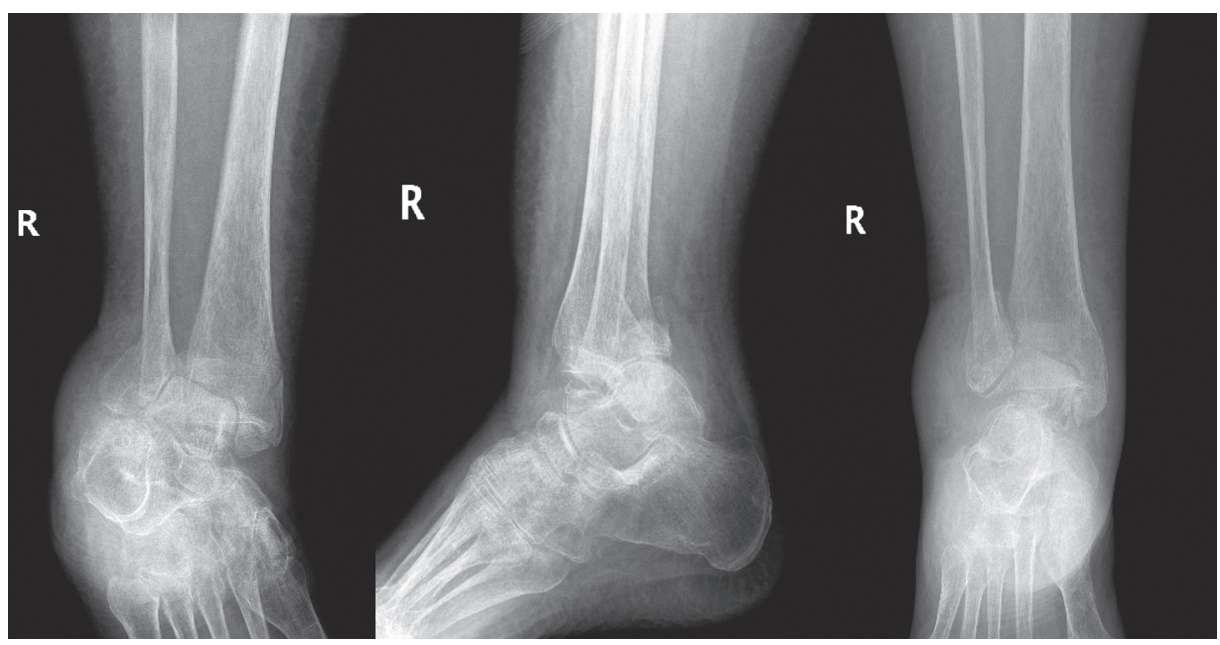

Fig. 1: X-rays demonstrating right ankle trimalleolar fracture dislocation

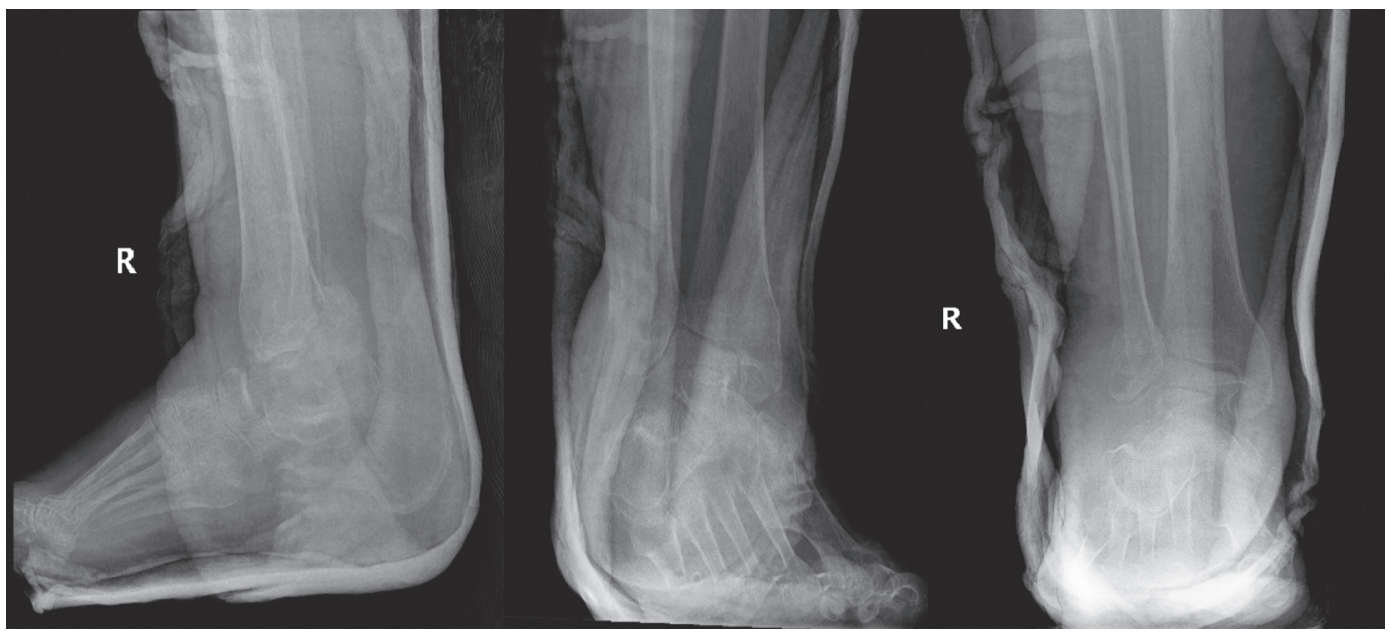

Fig. 2: X-rays demonstrating failed closed reduction of right ankle trimalleolar fracture dislocation

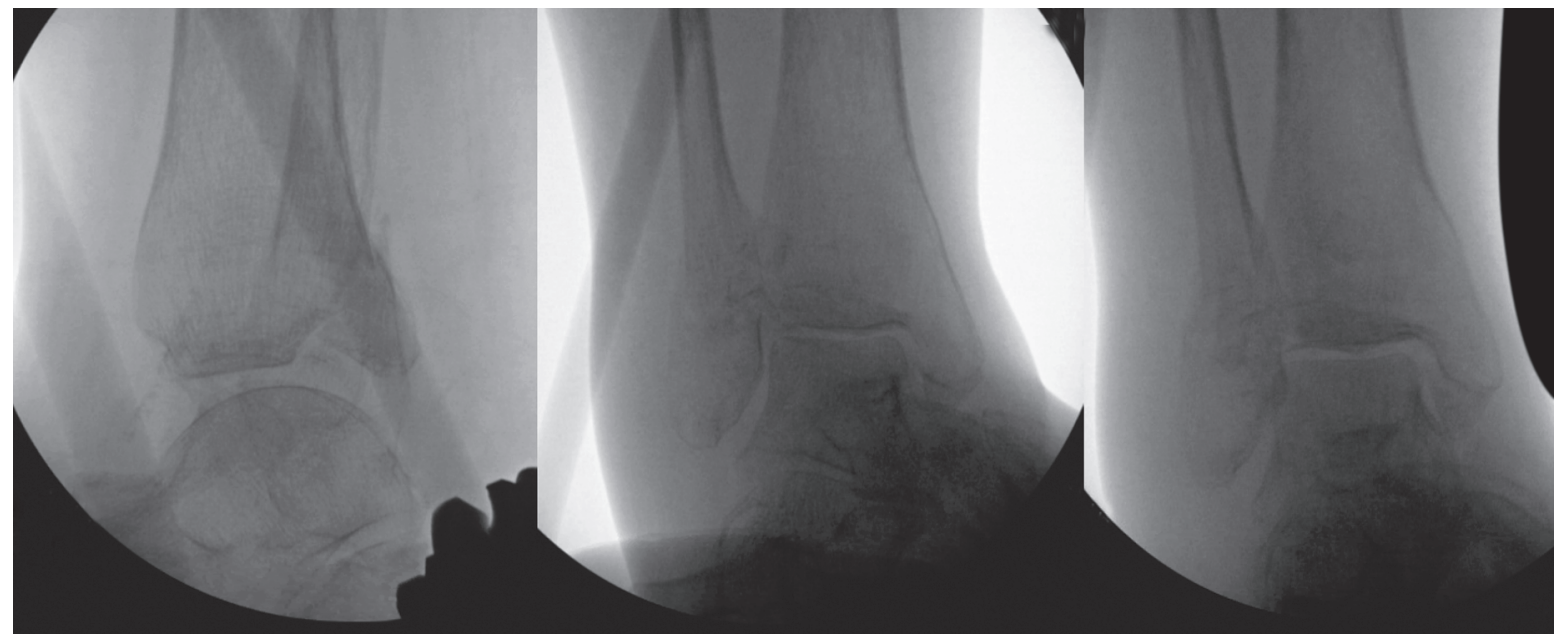

Fig. 3: Intraoperative images demonstrating closed reduction and placement of external fixation for right ankle trimalleolar fracture dislocation with appropriate alignment

(Fig. 3). The patient's hospital course was complicated by an exacerbation of her seizure disorder as well as a right femoral deep vein thrombosis (DVT). The patient was started on anticoagulant therapy, medically optimized, and ultimately discharged to rehab with a plan for definitive fixation once ankle soft tissue swelling had subsided.

The patient presented for an initial postoperative visit 2 weeks following external fixation, with $\mathrm{X}$-rays showing loss of reduction 
of her ankle fracture (Fig. 4). Given the patients' comorbidities and risks of surgery, the plan was to proceed with TTC arthrodesis with retrograde nail fixation. However, given the patients DVT, a delay in definitive fixation occurred as the patient required placement of an IVC filter before final fixation. Following IVC filter placement, 4 weeks following initial external fixation, the patient was scheduled for TTC arthrodesis, but the day before surgery the patient contracted COVID-19, which delayed her care even further.

After resolution, approximately 7 weeks post-injury, the patient presented for staged removal of the external fixator with a pin holiday followed by TTC arthrodesis using retrograde nail fixation. However, during external fixator removal, the patient was found to have a pin tract infection with calcaneal pin site cultures returning positive for Staph. epidermidis. Appropriate antibiotic therapy was started, and the patient was placed in a temporary short leg splint to allow for a pin holiday before final surgical fixation. To avoid contamination of the joint and intramedullary canal during TTC nail preparation, the decision was made to instead proceed with a posterior TTC plate arthrodesis. After a course of intravenous antibiotics, the case was performed through a single posterolateral incision used for both joint preparation and TTC plate application with AUGMENT Bone Graft (Wright Medical Technology, Inc, Memphis, TN) VIA ${ }^{\oplus}$ Graft (VIVEX Biologic, Miami, FI.), and BIO4
Viable Bone Matrix (Stryker, Portage, MI) used to augment the arthrodesis (Fig. 5).

\section{Discussion}

Successful management of high-risk diabetic patients with unstable ankle fractures remains a difficult task given the high incidence of complications and need for reoperation. Several options exist to help improve stability and reduce fixation failure including increased rigid fixation, combination internal and external fixation, and prolonged time to weight-bearing..$^{12}$ However, these strategies have not completely addressed the problem as complication and reoperation rates remain high. While primary TTC arthrodesis for ankle fractures is not a novel idea, techniques have evolved, and it has been proposed that primary TTC arthrodesis in high-risk diabetic patients can produce satisfactory results. ${ }^{14-17}$

Tibiotalocalcaneal arthrodesis can be achieved through various methods including TTC hindfoot nails, ringed external fixators, and TTC arthrodesis with crossed screws or locked plate constructs. Retrograde TTC nails have the advantage of being performed percutaneously through small incisions and be an effective primary treatment option for an ankle fracture in highrisk patients with complicated DM. ${ }^{15}$ However, this method often

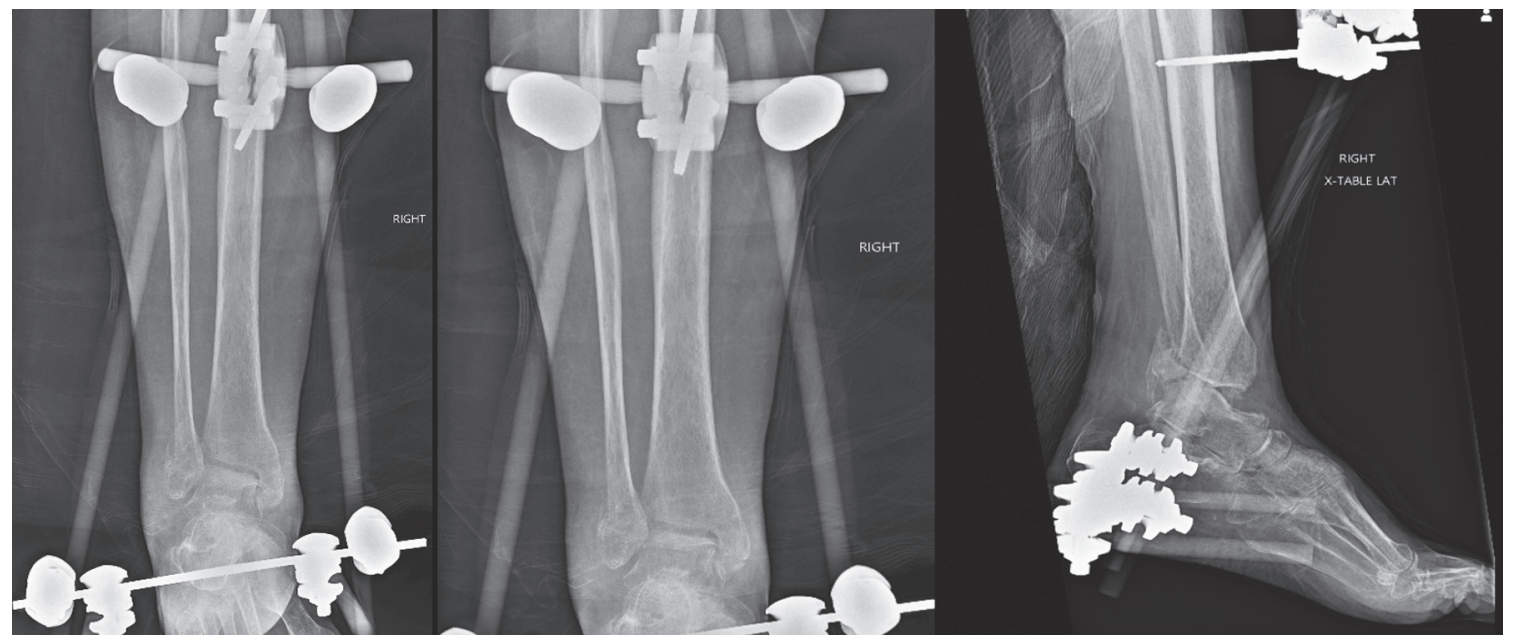

Fig. 4: Two weeks status post-external fixation right ankle trimalleolar fracture dislocation with loss of reduction

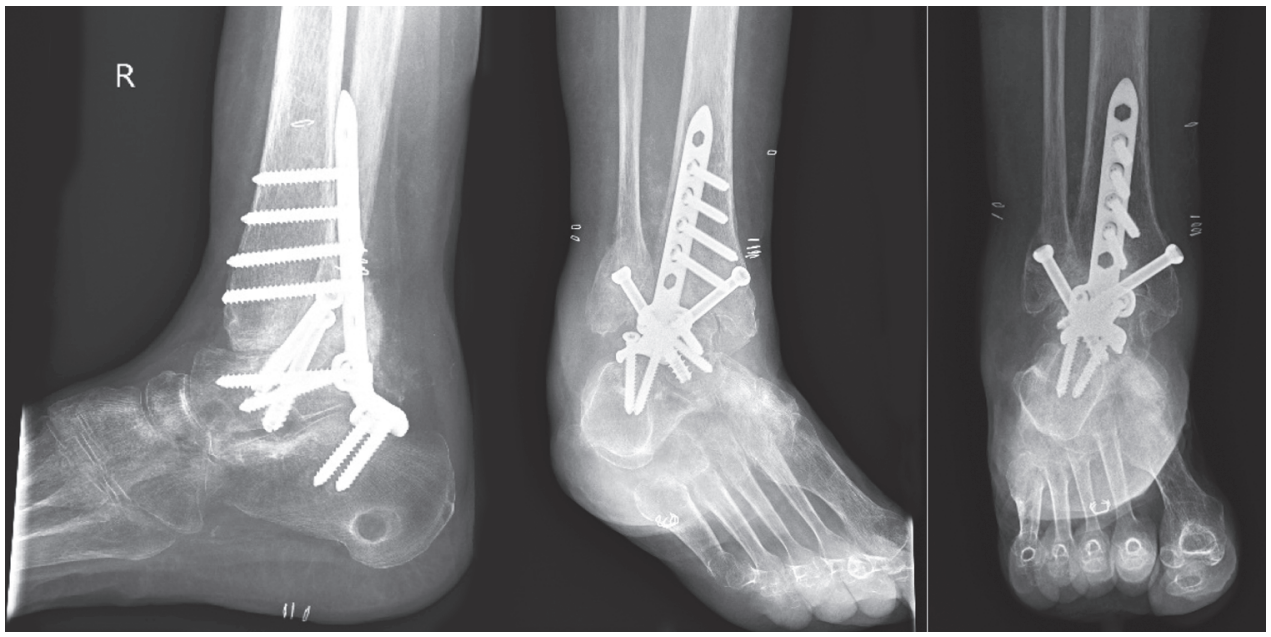

Fig. 5: X-rays right ankle status post-removal right ankle external fixation and TTC arthrodesis of right ankle trimalleolar fracture dislocation 
requires reaming which may increase the possibility of systemic inflammation, pulmonary embolism, and infection. Specifically, in the setting of infection, such as in our patient, a TTC fusion plate is preferred over retrograde TTC nails for ankle arthrodesis to avoid potentially seeding the intramedullary canal during hardware insertion. Furthermore, biomechanical studies confirmed that locked plate fixation had the best biomechanical fusion strength for TTC arthrodesis surgery, particularly for patients with poor bone quality, when compared to retrograde IMN fixation using cannulated compression screw nail and angle plate methods. ${ }^{18}$

Many plate designs exist for use of TTC arthrodesis, including anterior, posterior, and lateral plate constructs, which may be used to achieve stabilization in different configurations and alignments based on the surgeon's preference. Posterior and lateral plates were developed specifically to facilitate approaches that avoid high rates of wound complications and hardware irritation associated with anterior arthrodesis. ${ }^{19-22}$ In our patient, a posterior approach with a posterior TTC fusion plate was used to avoid hardware irritation and allow for adequate soft tissue coverage. However, regardless of approach, all the various plates utilize compression for the reduction of arthrodesis and provide stable internal fixation without the risks associated with external fixators. With TTC locking plate technology, the screw plate constructs provide rigid fixedangle fixation, increasing stability and load to failure compared with traditional ORIF constructs. This concept is particularly advantageous in patients with poor bone stock as locking plates have lower, irreversible deformation compared to intramedullary nail or blade plate fixation. ${ }^{23}$

To augment this slower-healing microenvironment, we utilized a combination of bone marrow aspirate and bone allograft for their osteogenic, osteoinductive, and osteoconductive properties. We specifically used biologics containing rhPDGF, VEGF, PDGF mixed with bone marrow aspirate and allograft containing demineralized cortical bone and mineralized cortical and cancellous bone. Both bone marrow aspirate containing concentrated amounts of progenitor cells and bone graft has been shown to improve fusion rates in higher risk patients. ${ }^{24,25}$ Bone graft has independently been shown to decrease the infection rate as well giving the added benefit in this susceptible group. ${ }^{26}$

Our case shows a TTC fusion plate is a safe and effective treatment option for unstable ankle fractures in high-risk diabetic patients. In comparison to previous studies assessing the use of traditional ORIF in related cohorts, the use of a TTC plate offers the advantages of rigid fixation, minimal soft tissue dissection, and relatively few complications. We were able to demonstrate that primary ankle arthrodesis with augmentation is effective in treating unstable ankle fractures, especially in the setting of diabetes and peripheral neuropathy.

\section{References}

1. Court-Brown CM, Caesar B. Epidemiology of adult fractures: a review. Injury 2006;37(8):691-697. DOI: 10.1016/j.injury.2006.04.130.

2. Schmidt T, Simske NM, Audet MA, et al. Effects of diabetes mellitus on functional outcomes and complications after torsional ankle fracture. J Am Acad Orthop Surg 2020;28(16):661-670. DOI: 10.5435/ JAAOS-D-19-00545.

3. Shibuya N, Davis ML, Jupiter DC. Epidemiology of foot and ankle fractures in the United States: an analysis of the National Trauma Data Bank (2007 to 2011). J Foot Ankle Surg 2014;53(5):606-608. DOI: 10.1053/j.jfas.2014.03.011.
4. Regan DK, Manoli 3rd A, Hutzler L, et al. Impact of diabetes mellitus on surgical quality measures after Ankle fracture surgery: implications for "Value-Based" compensation and "Pay for Performance". J Orthop Trauma 2015;29(12):e483-e486. DOI: 10.1097/ BOT.0000000000000394.

5. Bullard KM, Cowie CC, Lessem SE, et al. Prevalence of diagnosed diabetes in adults by diabetes type - United States, 2016. MMWR Morb Mortal Wkly Rep 2018;67(12):359-361. DOI: 10.15585/mmwr. mm6712a2.

6. Wukich DK, Joseph A, Ryan M, et al. Outcomes of ankle fractures in patients with uncomplicated versus complicated diabetes. Foot Ankle Int 2011;32(2):120-130. DOI: 10.3113/FAI.2011.0120.

7. Pincus $D$, Veljkovic $A$, Zochowski $T$, et al. Rate of and risk factors for intermediate-term reoperation after ankle fracture fixation: a population-based cohort study. J Orthop Trauma 2017;31(10):e315e320. DOI: 10.1097/BOT.0000000000000920.

8. Manway JM, Blazek CD, Burns PR. Special considerations in the management of diabetic ankle fractures. Curr Rev Musculoskelet Med 2018;11(3):445-455. DOI: 10.1007/s12178-018-9508-x.

9. Jones KB, Maiers-Yelden KA, Marsh JL, et al. Ankle fractures in patients with diabetes mellitus. J Bone Joint Surg Br 2005;87(4):489-495. DOI: 10.1302/0301-620X.87B4.15724.

10. Schon LC, Marks RM. The management of neuroarthropathic fracture-dislocations in the diabetic patient. Orthop Clin North Am 1995;26(2):375-392. DOI: 10.1016/S0030-5898(20)31999-4.

11. Perry MD, Taranow WS, Manoli 2nd A, et al. Salvage of failed neuropathic ankle fractures: use of large-fragment fibular plating and multiple syndesmotic screws. J Surg Orthop Adv 2005;14(2):85-91.

12. Chaudhary SB, Liporace FA, Gandhi A, et al. Complications of ankle fracture in patients with diabetes. J Am Acad Orthop Surg 2008;16(3):159-170. DOI: 10.5435/00124635-200803000-00007.

13. Ayoub MA. Ankle fractures in diabetic neuropathic arthropathy: can tibiotalar arthrodesis salvage the limb? J Bone Joint Surg $\mathrm{Br}$ 2008;90(7):906-914. DOI: 10.1302/0301-620X.90B7.20090.

14. Ebaugh MP, Umbel B, Goss D, et al. Outcomes of primary tibiotalocalcaneal nailing for complicated diabetic Ankle fractures. Foot Ankle Int 2019;40(12):1382-1387. DOI: 10.1177/1071100719869639.

15. Gougoulias N, Oshba H, Dimitroulias A, et al. Ankle fractures in diabetic patients. EFORT Open Rev 2020;5(8):457-463. DOI: 10.1302/2058-5241.5.200025.

16. Taylor BC, Hansen DC, Harrison R, et al. Primary retrograde tibiotalocalcaneal nailing for fragility ankle fractures. lowa Orthop J 2016;36:75-78.

17. Vaudreuil NJ, Fourman MS, Wukich DK. Limb salvage after failed initial operative management of bimalleolar ankle fractures in diabetic neuropathy. Foot Ankle Int 2017;38(3):248-254. DOI: 10.1177/1071100716676063.

18. O'Neill PJ, Logel KJ, Parks BG, et al. Rigidity comparison of locking plate and intramedullary fixation for tibiotalocalcaneal arthrodesis. Foot Ankle Int 2008;29(6):581-586. DOI: 10.3113/FAl.2008.0581.

19. Abebe E, Farrell DJ, Zelle B, et al. Primary posterior blade plate tibiotalar arthrodesis: a salvage procedure for complex nonreconstructable pilon fractures. J Orthop Trauma 2017;31(Suppl 3):S30-S33. DOI: 10.1097/BOT.0000000000000911.

20. Pellegrini MJ, Schiff AP, Adams Jr SB, et al. Outcomes of tibiotalocalcaneal arthrodesis through a posterior Achilles tendon-splitting approach. Foot Ankle Int 2016;37(3):312-319. DOI: 10.1177/1071100715615398.

21. Nickisch F, Avilucea FR, Beals T, et al. Open posterior approach for tibiotalar arthrodesis. Foot Ankle Clin 2011;16(1):103-114. DOI: 10.1016/j.fcl.2010.11.001.

22. Mulligan RP, Adams Jr SB, Easley ME, et al. Comparison of posterior approach with intramedullary nailing versus lateral transfibular approach with fixed-angle plating for tibiotalocalcaneal arthrodesis. Foot Ankle Int 2017;38(12):1343-1351. DOI: 10.1177/ 1071100717731728. 
23. Ahmad J, Pour AE, Raikin SM. The modified use of a proximal humeral locking plate for tibiotalocalcaneal arthrodesis. Foot Ankle Int 2007;28(9):977-983. DOI: 10.3113/FAI.2007.0977.

24. Hernigou P, Guissou I, Homma Y, et al. Percutaneous injection of bone marrow mesenchymal stem cells for ankle non-unions decreases complications in patients with diabetes. Int Orthop 2015;39(8):16391643. DOI: 10.1007/s00264-015-2738-2.
25. Dekker TJ, White P, Adams SB. Efficacy of a cellular allogeneic bone graft in foot and ankle arthrodesis procedures. Foot Ankle Clin 2016;21(4):855-861. DOI: 10.1016/j.fcl.2016.07.008.

26. Pitts C, Alexander B, Washington J, et al. Factors affecting the outcomes of tibiotalocalcaneal fusion. Bone Joint $J$ 2020;102-B(3):345-351. DOI: 10.1302/0301-620X.102B3.BJJ-20191325.R1. 